\title{
Prediction of Human Glomerular Filtration Rate from Preterm Neonates to Adults: Evaluation of Predictive Performance of Several Empirical Models
}

\author{
Iftekhar Mahmood $^{1,2}$ and Carl-Michael Staschen ${ }^{1}$
}

Received 23 August 2015; accepted 5 January 2016; published online 22 January 2016

\begin{abstract}
The objective of this study was to evaluate the predictive performance of several allometric empirical models (body weight dependent, age dependent, fixed exponent 0.75 , a data-dependent single exponent, and maturation models) to predict glomerular filtration rate (GFR) in preterm and term neonates, infants, children, and adults without any renal disease. In this analysis, the models were developed from GFR data obtained from inulin clearance (preterm neonates to adults; $n=93$ ) and the predictive performance of these models were evaluated in 335 subjects (preterm neonates to adults). The primary end point was the prediction of GFR from the empirical allometric models and the comparison of the predicted GFR with measured GFR. A prediction error within $\pm 30 \%$ was considered acceptable. Overall, the predictive performance of the four models (BDE, ADE, and two maturation models) for the prediction of mean GFR was good across all age groups but the prediction of GFR in individual healthy subjects especially in neonates and infants was erratic and may be clinically unacceptable.
\end{abstract}

KEY WORDS: allometry; GFR; pediatrics.

\section{INTRODUCTION}

Glomerular filtration rate (GFR) is widely used to characterize renal function across different age groups (preterm neonates to adults) and the knowledge of GFR is helpful for the adjustment of dose in children and adults for those drugs which are primarily eliminated by the renal route (1). GFR is generally determined by estimating the clearance of endogenous markers such as creatinine or cystatin- $\mathrm{C}$ and exogenously administered substances such as inulin, mannitol, iohexol, or ${ }^{51} \mathrm{Cr}$-ethylene diamine tetra-acetic acid $\left({ }^{51} \mathrm{Cr}\right.$ EDTA) (1). Besides determining GFR experimentally, equations with endogenous markers (such as Cockcroft-Gault and Schwartz formula) have been developed for everyday clinical use (1). These equations are intended to replace GFR (measured by inulin or creatinine clearance) as the use of these equations for GFR estimation may save time and effort.

Empirical approaches such as allometric scaling based on weight or age can be useful for the prediction of GFR across different age groups (from pre-term neonates to adults). Using the data of Rubin et al (2), Hayton (3) developed an allometric

The views expressed in this article are those of the authors and do not reflect the official policy of the FDA or any private enterprise. No official support or endorsement by the FDA or any private enterprise is intended or should be inferred.

${ }^{1}$ Division of Hematology Clinical Review Branch, Office of Blood Review \& Research (OBRR), Center for Biologic Evaluation and Research, Food \& Drug Administration, 10903 New Hampshire Avenue, Silver Spring, Maryland 20993-0002, USA.

${ }^{2}$ To whom correspondence should be addressed. (e-mail: Iftekhar.mahmood@fda.hhs.gov; ) model incorporating the maturation and growth of renal function that could be used to predict GFR in different age groups. Although Hayton suggested a fixed exponent of 0.662 for GFR, it is possible that a single exponent cannot describe the GFR data across all age groups (4). This phenomenon has been observed with the clearances of drugs when allometric models were developed across different age groups (4).

In recent years, in order to predict drug clearance in children, two empirical models known as maturation model and body weight-dependent allometric exponent (BDE) model have been proposed. The maturation model has been applied to predict clearance of several drugs such as morphine (5), propofol (6), and midazolam (7). The BDE model has been applied to predict clearance of morphine (8), propofol $(9,10)$, and busulfan (11). Besides BDE model, another empirical model called age-dependent exponent (ADE) model has been developed for the prediction of drug clearance from preterm neonates to adults (4). Both BDE and ADE models predict clearances of drugs based on allometric exponents which change with body weight or age.

Using the concept of maturation model, Rhodin et al (12) suggested an empirical maturation model to predict GFR in children and adults using weight and postmenstrual age. The authors pooled the data from different studies that included healthy subjects, premature neonates, and children. In these studies, the GFR was measured by different methods that included inulin, mannitol, polyfructose, iohexol, and ${ }^{51} \mathrm{Cr}-$ EDTA. Rhodin et al did not validate their maturation model by external data (data not used in the model) hence the predictive power of their model remains unknown. The ADE model has not been used to predict GFR across different age groups. 
The objectives of this study were to develop several empirical models from preterm to adults with normal renal function to predict GFR from pre-term neonates to adults. The description of these models is as follows:

- To develop BDE, ADE, and maturation models to predict GFR for different age groups.

- To develop two allometric models; one based on data-dependent allometric exponent and the other based on fixed exponent 0.75 .

- To evaluate the predictive performance of the empirical maturation model of Rhodin et al for GFR estimation using data that were not used by the authors in the development of their model (with the exception of one data set).

- To compare the predictive performance of all models for the prediction of GFR across different age groups by comparing predicted GFR by the models with experimentally determined GFR.

\section{METHODS}

\section{Development of the Models}

From the literature, GFR data measured by inulin clearance were obtained for different age groups. The subjects did not have any known renal disease or renal dysfunction. Models were developed from 93 subjects' GFR data. The following is the description of the data in the model development:

Preterm neonates $(n=20)$ : gestational age $=27-$ 37 weeks; chronological age $=0.3-4.2$ weeks; body weight $=0.68-2.25 \mathrm{~kg}$; GFR $=0.5-2.74 \mathrm{~mL} / \mathrm{min}(13)$;

Term neonates $(n=15)$ : gestational age $=38-42$ weeks; chronological age $=0.3-2$ weeks; body weight $=1.04$ $4.64 \mathrm{~kg}$; GFR = 1.38-8.4 $\mathrm{mL} / \mathrm{min}(14,15)$;

Infants $(n=18)$ : chronological age $=1.9-15$ months; body weight $=6-11 \mathrm{~kg}$; GFR = 6-37 $\mathrm{mL} / \mathrm{min}(16)$;

Children $(n=20)$ : chronological age $=2.75-16$ years; body weight $=13-51 \mathrm{~kg} ; \mathrm{GFR}=31-114 \mathrm{~mL} / \mathrm{min} ;$ and $(17,18)$

Adults $(n=20)$ : chronological age $=25-49$ years; body weight $=51-84 \mathrm{~kg} ;$ GFR $=87-167 \mathrm{~mL} / \mathrm{min}(19)$.

\section{Model 1: Body Weight-Dependent Exponent Model (BDE)}

The relationship between individual body weight $\left(\mathrm{BW}_{\mathrm{i}}\right)$ and individual GFR values $\left(\mathrm{GFR}_{\mathrm{i}}\right)$ of different age groups were described by Eq. 1.

$\mathrm{GFR}_{\mathrm{i}}=$ coefficient $\times\left(\mathrm{BW}_{\mathrm{i}} / 70 \mathrm{~kg}\right)^{\mathrm{L}} \times \mathrm{BW}_{\mathbf{i}^{(-\mathrm{M})}}$

Where, $\mathrm{L} \times \mathrm{BW}_{\mathrm{i}}^{-\mathrm{M}}$ defines the body weight-dependent exponent for GFR. The coefficient and the exponents $\mathrm{L}$ and $\mathrm{M}$ were estimated.

Individual reported GFR values from neonates to adults were analyzed by ADAPT 5 (User's Guide: Pharmacokinetic/ Pharmacodynamic Systems Analysis Software. Biomedical Simulations Resource, Los Angeles, 2009) using the maximum likelihood estimator. Graphic plots were prepared using Prism (Version 6.01, GraphPad Software Inc, La Jolla, CA).

\section{Model 2: Age-Dependent Exponent Model (ADE)}

In this method, different exponents were used for different age groups and GFR was predicted in a given age group according to Eq. 2.

GFR in an individual $=$ Adult GFR $\times\left(\mathrm{W}_{\mathrm{C}} / 70\right)^{\mathrm{b}}$

Where the "adult GFR" is the mean adult GFR $(120 \mathrm{~mL} /$ $\min ) . W_{C}$ is the weight of a child and 70 is a standardized adult body weight. In order to predict GFR in adults, actual adult body weight was divided by $70 \mathrm{~kg}$ in Eq. 2 .

Exponent "b" in Eq. 2 is age dependent. The exponents used in this method were $1.15,1.0,0.9$, and 0.75 for preterm neonates, term neonates, $>6$ months to 1 year, and $>1$ year, respectively. The exponents selected in the ADE model are based from previous experience with the prediction of drug clearance across different age groups, exponents from BDE model analyzed in this study, observation, and data analysis. The range of exponents in the ADE model is arbitrary and was selected based on the suitability of the exponents to predict GFR values as close to the observed GFR values.

\section{Model 3: Allometric Model (Fixed Exponent 0.75)}

The GFR was predicted in individual subject by using a fixed exponent of 0.75 on body weight as shown in Eq. 3. The denominator refers to a standard adult body weight of $70 \mathrm{~kg}$

GFR in an individual $=$ Adult GFR $\times\left(\mathrm{W}_{\mathrm{C}} / 70\right)^{0.75}$

Where the "adult GFR" is the mean adult GFR $(120 \mathrm{~mL} /$ $\min ) . \mathrm{W}_{\mathrm{C}}$ is the weight of a child and $\mathrm{W}_{\mathrm{A}}$ is the weight of an adult standardized to $70 \mathrm{~kg}$. In order to predict GFR in adults, actual adult body weight was divided by $70 \mathrm{~kg}$ in Eq. 3 .

\section{Model 4: Allometric Model (Data-Dependent Exponent)}

This model was developed using the GFR and body weights from preterm neonates to adults. In this model, GFR values were plotted against body weights $/ 70 \mathrm{~kg}$ of different age groups on a log-log scale and Eq. 4 was then used to predict GFR across different age groups.

$\mathrm{GFR}$ in an individual $=\mathrm{a} \times(\mathrm{BW} / 70)^{\mathrm{b}}$

Where " $a$ " is the coefficient and " $b$ " is the exponent of the allometric equation.

Model 4 differs from models 1 and 2 with respect to exponents. Models 1 and 2 assume that the exponents of allometry vary depending on weight or age whereas model 4 assumes that a single exponent can describe the entire data across all age groups. Model 4 also differs from model 3 with respect to exponent since model 3 assumes that GFR can be estimated from a fixed exponent of 0.75 across all age groups. 


\section{Model 5: Maturation Model}

Equation 5 describes a maturation model that incorporates both weight $(\mathrm{BW})$ and age.

$$
\begin{gathered}
\text { GFR in an individual }=\mathrm{GFR}_{\text {std }} \times\left(\frac{\mathrm{BW}}{70 \mathrm{~kg}}\right)^{0.75} \\
\frac{(\mathrm{PMA})^{\text {HillGFR }}}{\left(\mathrm{PMA}_{50}\right)^{\mathrm{HillGFR}}+(\mathrm{PMA})^{\text {HillGFR }}}
\end{gathered}
$$

Where $\mathrm{GFR}_{\text {std }}$ is the population estimate for GFR, BW is the individual body weight, and 0.75 represents a fixed allometric exponent on body weight. PMA is the postmenstrual age in weeks, $\mathrm{PMA}_{50}$ is the PMA at which normalized clearance is equal to $50 \%$ of the maximum value, and Hill $_{\mathrm{GFR}}$ is an exponent that describes the steepness of the maturation function.

Individual reported GFR values from neonates to adults were normalized to $(\mathrm{BW} / 70 \mathrm{~kg})^{0.75}$. The parameter estimates of the right hand side of Eq. 5 (GFR $_{\text {std }}, \mathrm{PMA}_{50}$, and Hill $\left.\mathrm{GFR}_{\mathrm{G}}\right)$ were obtained by fitting the body weight normalized GFR to the maturation function. Data were analyzed by ADAPT 5 (User's Guide: Pharmacokinetic/Pharmacodynamic Systems Analysis Software. Biomedical Simulations Resource, Los Angeles, 2009) using the maximum likelihood. Graphic plots were prepared using Prism (Version 6.01, GraphPad Software Inc, La Jolla, CA). We compared the predictive power of our model with the model of Rhodin et al. It should be noted that the model of Rhodin et al was developed from 923 subjects and included GFR values obtained from different methods that included inulin, mannitol, polyfructose, iohexol, and ${ }^{51} \mathrm{Cr}$-EDTA.

\section{Validation of the Models}

Models 1-5 were validated with external data (not included in the model) for several age groups. Although, in this study, the models were developed from inulin clearance only, the models were also validated for creatinine clearance (CRCL). The description of the validation data $(n=335)$ are as follows:

Inulin Preterm $(n=109)$ (references $(6,20-22))$; term $(n=29)$ $(21,22)$; children (4-11.3 years; $n=17)(17,18)$; adolescents $(12-15$ years; $n=14)(18,23,24)$; adults (19-49 years; $n=34(19,23,24)$; total $=203$ subjects

CRCL preterm $(n=29)(25,26)$; term $(n=31)(14,25,26)$; infants (7-113 days; $n=12)(27)$; children (3.312 years; $n=21)(28-30)$; adolescents (13-17 years; $n=6)(29,30)$; adults $(20-39$ years; $n=33)(31-34)$; total $=132$ subjects.

Although Coulthard's (13) data were used by Rhodin et al in the development of their model, data from Coulthard (preterm neonates) were used in the validation of all models because these data set helped to increase the sample size for the validation. In this study, Coulthard's data were not used in the development of any model.

\section{STATISTICAL ANALYSIS}

Percent error between the observed and predicted values was calculated according to the following equation:

$\%$ error $=\frac{(\text { predicted }- \text { observed }) \times 100}{\text { observed }}$

The bias of the methods was measured by calculating the mean prediction error (MPE) according to the following Eq. (7):

$\operatorname{MPE}=\frac{\sum(\text { predicted }- \text { observed })}{n}$

Where $n$ is the number of observations.

The precision of the methods was measured by calculating the root mean square error (RMSE) according to the following Eq. (8):

Mean Square Error $(\mathrm{MSE})=\frac{\sum(\text { Predicted-observed })^{2}}{n}$

$\mathrm{RMSE}=(\mathrm{MSE}) 0.5$

Both MPE and RMSE were expressed as percent of mean using Eq. 10:

$\% \mathrm{MPE}$ or RMSE $=\frac{(\mathrm{MPE} \text { or RMSE } \times 100)}{\text { mean observed CL }}$

Further assessment of the suitability of the methods was done by grouping the number of subjects for each age group according to \%error; from $\leq 10$ to $\leq 30 \%(35,36)$.

\section{RESULTS}

The observed and predicted GFR values using inulin and CRCL by different models for different age groups, bias and precision of the prediction and the percent of subjects within 10 or $30 \%$ prediction error are summarized in Tables I, II, III, and IV. The GFR models were validated in a total of 335 subjects of which there were 203 subjects for inulin clearance and 132 subjects for CRCL.

GFR was predicted in an individual subject but the results are presented as mean GFR in a given age group. The mean predicted GFR values were in good agreement with the observed GFR values for all models (exception being exponent 0.75 for preterm and term neonates). Mean GFR values, however, are of no practical value; hence, the focus was on individual GFR prediction with especial emphasis on preterm and term neonates and younger children. Individual predicted GFR values, in most of the instances, were erratic, and were not in agreement with the observed GFR values. Individual prediction error of GFR ranged from $0 \%$ to more 
Table I. Observed and Predicted ( \pm sd) GFR Based on Inulin Clearance by Different Models Across Several Age Groups

\begin{tabular}{|c|c|c|c|c|c|}
\hline Models & Preterm $(n=103)$ & Term $(n=29)$ & Children $(n=17)$ & Adolescents $(n=14)$ & Adults $(n=34)$ \\
\hline Observed & $1.8 \pm 1.1$ & $4.5 \pm 2.5$ & $76 \pm 17$ & $102 \pm 15$ & $114 \pm 22$ \\
\hline Model 1 & $1.7 \pm 1.1$ & $4.6 \pm 2.2$ & $61 \pm 19$ & $99 \pm 17$ & $120 \pm 9$ \\
\hline Model 2 & $1.4 \pm 0.6$ & $4.6 \pm 1.4$ & $56 \pm 15$ & $90 \pm 17$ & $111 \pm 9$ \\
\hline Model 3 & $6.4 \pm 1.7$ & $10.3 \pm 2.4$ & $56 \pm 15$ & $90 \pm 17$ & $111 \pm 9$ \\
\hline Model 4 & $1.8 \pm 0.8$ & $3.8 \pm 1.4$ & $60 \pm 26$ & $125 \pm 38$ & $172 \pm 23$ \\
\hline Maturation 1 & $1.7 \pm 0.7$ & $3.8 \pm 0.9$ & $66 \pm 18$ & $105 \pm 20$ & $130 \pm 11$ \\
\hline Maturation 2 & $1.4 \pm 0.7$ & $3.8 \pm 1.0$ & $57 \pm 16$ & $91 \pm 17$ & $112 \pm 22$ \\
\hline
\end{tabular}

Model 1 = body weight-dependent exponent model (BDE); Model 2 = age-dependent exponent model (ADE); Model $3=$ allometric model (fixed exponent 0.75); Model 4 = allometric model (data-dependent exponent); Maturation model $1=$ this study, Maturation model $2=$ Rhodin et al study

than $100 \%$ from all models with uncertainty in individual prediction. In this analysis, a prediction error of $\leq 30 \%$ for individual GFR was considered acceptable $(35,36)$. The results of the study for the individual models are summarized below.

\section{Model 1: Body Weight-Dependent Exponent Model (BDE)}

Estimated BDE model parameters (coefficients and exponents $\mathrm{L}$ and $\mathrm{M}$ ) and percent coefficient of variation (\%CV) for GFR from inulin clearance data were as follows:

$$
\begin{aligned}
\text { Coefficient } & =128 \mathrm{~mL} / \min (\% \mathrm{CV}=7) ; \mathrm{L} \\
& =1.199(\% \mathrm{CV}=2) ; \mathrm{M} \\
& =-0.157(\% \mathrm{CV}=14) .
\end{aligned}
$$

Prediction of GFR based on inulin clearance The mean $( \pm$ sd) exponents of allometry from BDE model for preterm neonates, term neonates, children, adolescents, and adults were $1.15 \pm 0.06,1.04 \pm 0.05,0.73 \pm 0.04,0.66 \pm 0.03$, and 0.63 \pm 0.01 , respectively. The exponents of allometry followed the general pattern seen with the BDE models for the prediction of drug clearance and are allometrically meaningful since the exponents of allometry for drug clearance vary with age.

The mean predicted and observed GFR values in different age groups from BDE are summarized in Table I. Figure 1 represents a BDE model of GFR (from preterm neonates to adults). The mean predicted GFR values in all age groups by the BDE model were in good agreement with the observed mean GFR values. The bias and precision of the BDE model are shown in Table II. With increasing age, the bias in the BDE models' predictive power decreased and the precision improved. The individual prediction of GFR was erratic, especially in preterm and term neonates. In preterm neonates, $41 \%$ subjects had a prediction error of $\leq 30 \%$, whereas in term neonates, there were only $31 \%$ subjects with less than $\leq 30 \%$ prediction error (Table III). In children, adolescents, and adults, more than $75 \%$ of individuals had prediction error $\leq 30 \%$ (Table III). The number of subjects with $\leq 10 \%$ prediction error in each age group was less than the number of subjects with prediction error of $\leq 30 \%$.

Prediction of GFR based on creatinine clearance The BDE model developed from inulin clearance was tested with the GFR measured from CRCL. The mean $( \pm \mathrm{sd})$ exponents of allometry from BDE model for preterm neonates, term neonates, infants, children, adolescents, and adults were $1.10 \pm 0.07,1.01 \pm 0.03,0.99 \pm 0.03,0.73 \pm 0.04,0.68 \pm 0.02$, and $0.61 \pm 0.01$, respectively.

The mean predicted and observed GFR based on CRCL in different age groups from BDE are summarized in Table II. The mean predicted GFR values in all age groups by the BDE model were in good agreement with the observed mean GFR values. The bias and precision of the BDE model are shown in Table IV. As seen with the prediction of GFR based on inulin clearance, with increasing age bias in the BDE models' predictive power decreased and the precision improved. The individual prediction of GFR was erratic, especially in preterm and term neonates. In preterm neonates, approximately $48 \%$ subjects had a prediction error of

\begin{tabular}{|c|c|c|c|c|c|c|}
\hline Models & Preterm $(n=29)$ & Term $(n=31)$ & Infants $(n=12)$ & Children $(n=21)$ & Adolescents $(n=6)$ & Adults $(n=33)$ \\
\hline Observed & $2.1 \pm 1.0$ & $5.1 \pm 2.2$ & $5.8 \pm 2.1$ & $58 \pm 26$ & $79 \pm 27$ & $130 \pm 31$ \\
\hline Model 1 & $2.7 \pm 0.7$ & $5.6 \pm 1.5$ & $6.5 \pm 2.0$ & $59 \pm 18$ & $82 \pm 12$ & $130 \pm 11$ \\
\hline Model 2 & $1.9 \pm 0.7$ & $5.3 \pm 0.9$ & $5.8 \pm 1.3$ & $54 \pm 14$ & $74 \pm 11$ & $123 \pm 13$ \\
\hline Model 3 & $8.0 \pm 2.0$ & $11.6 \pm 1.6$ & $12.4 \pm 2.0$ & $54 \pm 14$ & $74 \pm 11$ & $123 \pm 13$ \\
\hline Model 4 & $2.5 \pm 1.0$ & $4.5 \pm 1.0$ & $5.0 \pm 1.3$ & $56 \pm 24$ & $89 \pm 22$ & $202 \pm 34$ \\
\hline Maturation 1 & $2.1 \pm 0.8$ & $4.1 \pm 0.6$ & $5.8 \pm 1.7$ & $63 \pm 18$ & $86 \pm 13$ & $144 \pm 14$ \\
\hline Maturation 2 & $1.8 \pm 0.7$ & $4.0 \pm 0.7$ & $6.3 \pm 2.1$ & $55 \pm 15$ & $74 \pm 11$ & $123 \pm 12$ \\
\hline
\end{tabular}
$\leq 30 \%$, whereas in term neonates, there were about $52 \%$

Table II. Observed and Predicted ( \pm sd) GFR Based on Creatinine Clearance by Different Models Across Several Age Groups

Model 1 = body weight-dependent exponent model (BDE); Model 2 = age-dependent exponent model (ADE); Model 3 = allometric model (fixed exponent 0.75); Model 4 = allometric model (data dependent exponent); Maturation model $1=$ this study, Maturation model $2=$ Rhodin et al study 
Table III. Bias and \%RMSE and Percent of Subjects Within $\leq 10$ and $\leq 30$ Prediction Error Based on Inulin Clearance

\begin{tabular}{|c|c|c|c|c|c|c|c|c|c|c|c|c|}
\hline & \multicolumn{6}{|c|}{$\%$ Bias } & \multicolumn{6}{|c|}{$\%$ RMSE } \\
\hline & $\mathrm{BDE}$ & $\mathrm{ADE}$ & Е 0.75 & Allom & Mat 1 & Mat 2 & $\mathrm{BDE}$ & $\mathrm{ADE}$ & Е 0.75 & Allom & Mat 1 & Mat 2 \\
\hline Preterm & 5 & 21 & -266 & -2 & 6 & 23 & 62 & 57 & 280 & 55 & 55 & 60 \\
\hline Term & -1 & -2 & -129 & 16 & 14 & 15 & 53 & 48 & 140 & 51 & 50 & 51 \\
\hline Children & 19 & 25 & 25 & 21 & 13 & 25 & 29 & 32 & 32 & 43 & 25 & 32 \\
\hline Adolescents & 3 & 12 & 12 & -22 & -3 & 11 & 21 & 23 & 23 & 42 & 23 & 23 \\
\hline \multirow[t]{2}{*}{ Adults } & -5 & 3 & 3 & -50 & -13 & 2 & 21 & 21 & 21 & 57 & 25 & 21 \\
\hline & \multicolumn{6}{|c|}{ Percent subjects with $\leq 30$ prediction error } & \multicolumn{6}{|c|}{ Percent subjects with $\leq 10$ prediction error } \\
\hline Preterm & 41 & 58 & 0 & 51 & 48 & 47 & 19 & 27 & 0 & 16 & 20 & 17 \\
\hline Term & 31 & 35 & 3 & 38 & 45 & 41 & 17 & 7 & 0 & 10 & 10 & 10 \\
\hline Children & 77 & 71 & 71 & 71 & 77 & 71 & 24 & 18 & 18 & 24 & 35 & 18 \\
\hline Adolescents & 86 & 86 & 86 & 64 & 100 & 86 & 21 & 43 & 43 & 14 & 29 & 43 \\
\hline Adults & 88 & 91 & 91 & 24 & 68 & 91 & 38 & 29 & 29 & 15 & 18 & 29 \\
\hline
\end{tabular}

$B D E$ body weight-dependent exponent model (BDE), $A D E$ age-dependent exponent model (ADE), E 0.75 exponent 0.75 , Allom allometric model, Mat 1 this study, Mat 2 Rhodin et al maturation model

subjects with less than $\leq 30 \%$ prediction error (Table IV). In children, adolescents, and adults, more than $65 \%$ of individuals had prediction error $\leq 30 \%$ (Table IV).

Overall, from the BDE model, predicted mean GFR from preterm neonates to adults was comparable for all age groups but individual prediction was erratic especially in preterm and term neonates. Even in children, adolescents, and adults, the prediction error of $>30 \%$ was observed in about $20-25 \%$ subjects.

\section{Model 2: Age-Dependent Exponent Model (ADE)}

Unlike fixed exponent of 0.75 (model 3) or single exponent allometry, ADE uses different exponents for different age groups. The exponents used in this method were $1.15,1.0,0.9$, and 0.75 for preterm neonates, term neonates, $>6$ months to 1 year, and $>1$ year, respectively. Both in terms of mean and individual prediction of GFR, ADE predictive performance was far superior to the fixed exponent of 0.75 in preterm and term neonates as well as in children at least till 1 year of age.

Prediction of GFR based on inulin clearance The mean predicted and observed GFR values in different age groups from ADE model are summarized in Table I. The mean predicted GFR values in all age groups by the ADE model were in good agreement with the observed mean GFR values. The bias and precision of the ADE model are shown in Table II. In preterm neonates, approximately 58\% subjects had a prediction error of $\leq 30 \%$, whereas in term neonates, there were only $35 \%$ subjects with less than $\leq 30 \%$ prediction error (Table I). In children, adolescents, and adults, more than $70 \%$ of individuals had prediction error $\leq 30 \%$ (Table II).

Prediction of GFR based on creatinine clearance The mean predicted and observed GFR values in different age groups from ADE model are summarized in Table II. The mean predicted GFR values in all age groups by the ADE model

Table IV. Bias and \%RMSE and Percent of Subjects Within $\leq 10$ and $\leq 30$ Prediction Error Based on Creatinine Clearance

\begin{tabular}{|c|c|c|c|c|c|c|c|c|c|c|c|c|}
\hline & \multicolumn{6}{|c|}{$\%$ Bias } & \multicolumn{6}{|c|}{ \%RMSE } \\
\hline & $\mathrm{BDE}$ & $\mathrm{ADE}$ & Е 0.75 & Allom & Mat 1 & Mat 2 & $\mathrm{BDE}$ & $\mathrm{ADE}$ & Е 0.75 & Allom & Mat 1 & Mat 2 \\
\hline Preterm & -29 & 8 & -280 & -20 & 2 & 14 & 49 & 28 & 287 & 35 & 29 & 33 \\
\hline Term & -10 & -4 & -125 & 13 & 20 & 22 & 44 & 40 & 132 & 42 & 44 & 45 \\
\hline Infants & -13 & -2 & -116 & 12 & -2 & -10 & 35 & 30 & 120 & 32 & 24 & 26 \\
\hline Children & -2 & 6 & 6 & 3 & -9 & 5 & 26 & 28 & 28 & 24 & 27 & 28 \\
\hline Adolescents & -3 & 7 & 7 & -13 & -8 & 4 & 30 & 30 & 30 & 35 & 25 & 24 \\
\hline \multirow[t]{2}{*}{ Adults } & 1 & 6 & 6 & -55 & -11 & 4 & 20 & 20 & 20 & 59 & 22 & 20 \\
\hline & \multicolumn{6}{|c|}{ Percent subjects with $\leq 30$ prediction error } & \multicolumn{6}{|c|}{ Percent subjects with $\leq 10$ prediction error } \\
\hline Preterm & 48 & 66 & 0 & 59 & 72 & 55 & 17 & 21 & 0 & 31 & 14 & 21 \\
\hline Term & 52 & 45 & 0 & 48 & 42 & 36 & 16 & 26 & 0 & 7 & 13 & 3 \\
\hline Infants & 50 & 67 & 8 & 75 & 75 & 67 & 25 & 17 & 0 & 17 & 25 & 25 \\
\hline Children & 81 & 76 & 76 & 71 & 71 & 76 & 24 & 29 & 29 & 29 & 24 & 29 \\
\hline Adolescents & 67 & 50 & 50 & 67 & 67 & 50 & 33 & 0 & 0 & 17 & 33 & 0 \\
\hline Adults & 88 & 91 & 91 & 18 & 76 & 91 & 36 & 42 & 42 & 3 & 27 & 42 \\
\hline
\end{tabular}

$B D E$ body weight dependent exponent model (BDE), ADE age-dependent exponent model (ADE), E 0.75 exponent 0.75, Allom allometric model, Mat 1 this study, Mat 2 Rhodin et al maturation model 


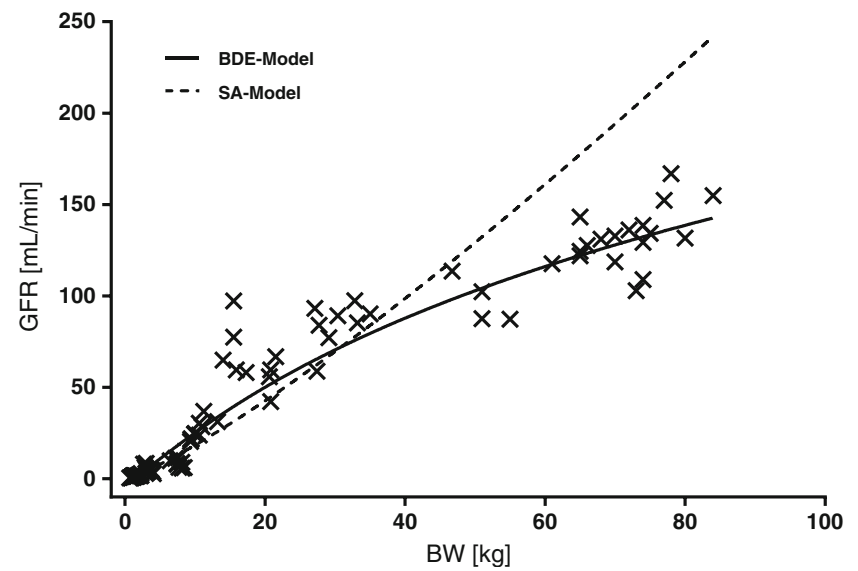

Fig. 1. Observed (symbol $x$ ) and predicted glomerular filtration rate (GFR, $\mathrm{mL} / \mathrm{min}$ ) plotted against body weight $(\mathrm{kg})$. The solid line represents the best fit to the body weight-dependent allometric exponent (BDE) model. The broken line demonstrates the best fit to the simple allometric (SA) model

were in good agreement with the observed mean GFR values. The bias and precision of the BDE model are shown in Table IV. With increasing age, bias in the ADE models' predictive power decreased and the precision improved. In preterm neonates, approximately $66 \%$ subjects had a prediction error of $\leq 30 \%$, whereas in term neonates, there were about $45 \%$ subjects with less than $\leq 30 \%$ prediction error (Table IV). In children and adults, more than $75 \%$ of individuals had prediction error $\leq 30 \%$ (Table IV).

\section{Model 3: Allometric Model (Fixed Exponent 0.75)}

Unlike ADE (model 2) and the data-dependent allometric models (model 4), this method uses a fixed exponent of 0.75 for the prediction of GFR, irrespective of age. The mean predicted and observed GFR values based on inulin and creatinine clearance are shown in Tables I and II, respectively. The mean predicted as well as individual GFR predicted values from this model were highly erratic (overestimated) in preterm, term, and infants. In these age groups, bias in the prediction was substantial and the precision was poor. In these three age groups, the prediction error of $\leq 30 \%$ was observed in only one subject. This model's predictive power was comparable with other models for children, adolescents, and adults.

\section{Model 4: Allometric Model (Data-Dependent Exponent)}

The coefficients and exponents of the allometric model were estimated to $194 \mathrm{~mL} / \mathrm{min}(\% \mathrm{CV}=8)$ and $1.21(\% \mathrm{CV}=2)$, respectively. Figure 1 represents a single-exponent allometric model of GFR (from preterm neonates to adults). The objective of the development of this model was to demonstrate that a single exponent does not describe GFR data across all age groups and more realistic models are BDE and ADE models (models 1 and 2). From Fig. 1, it is obvious that GFR will be overestimated at least in adults and this was noted with the model validation (Tables I and II). The mean predicted and observed GFR values in different age groups from this model are shown in Tables I and II, respectively. Overall, in preterm and term neonates, this model's predictive power was comparable with the BDE model (as well as other models) but as the age increased the models predictive power decreased. In adults, the GFR predicted by model 4 was substantially high with high bias and low precision as compared to other models. Based on inulin clearance and CRCL, the prediction error of $\leq 30 \%$ (individual subjects) in adults was observed in 24 and $18 \%$ subjects, respectively (Tables II and IV).

\section{Models 5: Maturation Models}

In order to predict GFR from preterm neonates to adults, two maturation models were used. We developed a maturation model from 93 subjects GFR data obtained from inulin clearance. We compared our maturation model from the maturation model developed by Rhodin et al.

Estimated maturation model parameters $\left(\mathrm{CL}_{\mathrm{std}}, \mathrm{PMA}_{50}\right.$, and Hill coefficient) and \% CV from this study and the model of Rhodin et al were as follows:

$$
\begin{aligned}
& \text { Maturation model (this study) }=\mathrm{CL}_{\text {std }} \\
& =141 \mathrm{~mL} / \mathrm{min}(\% \mathrm{CV}=5), \mathrm{PMA}_{50} \\
& =57.8 \text { weeks }(\% \mathrm{CV}=8), \text { Hill coefficient } \\
& =2.17(\% \mathrm{CV}=11)
\end{aligned}
$$

$$
\begin{aligned}
& \text { Rhodin } \text { et al } \text { model }=\mathrm{CL}_{\text {std }}=121 \mathrm{~mL} / \mathrm{min}, \mathrm{PMA}_{50} \\
& \quad=47.7 \text { weeks, Hill coefficient }=3.4
\end{aligned}
$$

The parameters of the two maturation models are different and this is not surprising because the parameters of a maturation model are data dependent and physiologically irrelevant.

Prediction of GFR based on inulin clearance The mean predicted and observed GFR values in different age groups from both maturation models are summarized in Table I. Figure 2 represents our maturation model of GFR (from preterm neonates to adults). The mean predicted GFR values in all age groups from both maturation models were in good agreement with the observed mean GFR values. The bias and precision of both maturation models are shown in Table II. As seen with other models, with increasing age bias in both maturation models predictive power decreased and the precision improved. The individual prediction of GFR was erratic, especially in preterm and term neonates (Table II). In preterm neonates, approximately $47-48 \%$ subjects had a prediction error of $\leq 30 \%$, whereas in term neonates, there were about $41-45 \%$ subjects with $\leq 30 \%$ prediction error (Table II). In children, adolescents, and adults, more than $70 \%$ of individuals had prediction error $\leq 30 \%$ (Table II). Both maturation models followed the same pattern as seen 


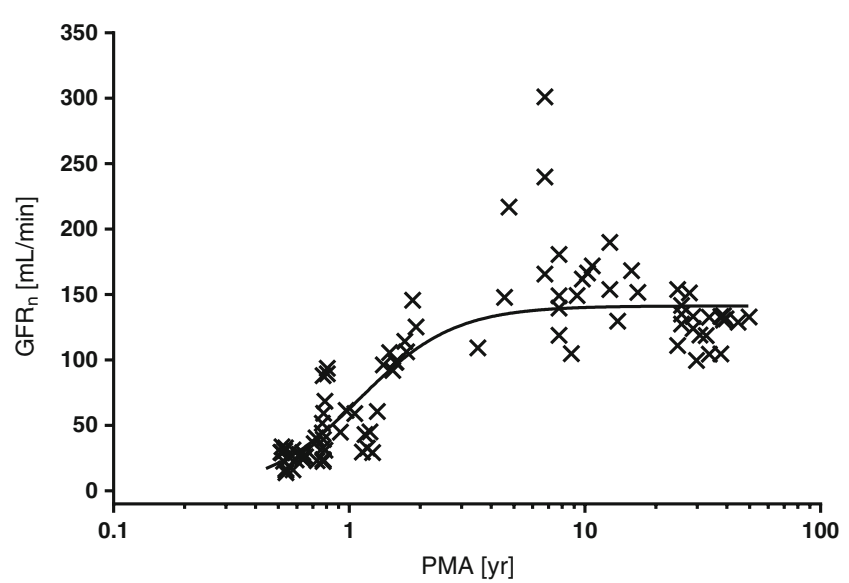

Fig. 2. Semi-logarithmic plot of observed (symbol $x$ ) and predicted glomerular filtration rate $\left[\mathrm{GFR}_{\mathrm{n}}, \mathrm{mL} / \mathrm{min}\right.$, standardized to body weight $\left(\mathrm{BW}_{\mathrm{i}} / 70 \mathrm{~kg}\right)^{0.75}$ ] plotted against post-menstrual age (PMA) in years $(\mathrm{yr})$. The solid line represents the best fit to the nonlinear maturation model

with ADE and BDE models and were comparable with ADE and BDE models in terms of their predictive power.

Prediction of GFR based on creatinine clearance The predictive performance of both maturation models were comparable and followed a similar pattern as seen with models 1 and 2 (Tables I, II, III, and IV). Model 1 has higher bias and lower precision than the two maturation models for preterm neonates. In preterm neonates, the number of subjects with prediction error of $\leq 30 \%$ by the BDE model was much lower than model 2 and the two maturation models. On the other hand, in term neonates, the BDE model performed much better than model 2 and the two maturation models. This indicates uncertainty in the predictive power of the models.

\section{DISCUSSION}

The knowledge of GFR can be important for the selection of a suitable dose for exclusively renally excreted drugs. One can determine GFR in a subject either by exogenous or endogenous markers or can estimate by one of the several formulae suggested by many investigators $(1,37)$. However, determination of GFR by these markers can be time consuming and technically difficult. Administration of inulin or any other exogenous substance to neonates and young children to determine GFR can be much more difficult than older children and adults. Therefore, formulae or models can be very useful in the determination of GFR in a given population, especially in neonates and young children. Furthermore, it is important to establish the predictive performance of these formulae or models and the prediction error of these formulae or models must be clinically acceptable.

In this study, we developed two new models namely BDE and ADE models to predict GFR from preterm neonates to adults. Both these models have been proposed for the prediction of drug clearances in pediatrics $(4,8-11)$.
Although the BDE model was developed based on inulin clearance, the model's predictive power was comparable for GFR based on inulin and creatinine clearance. The BDE model was developed based on only 93 subjects' data, yet its predictive power was comparable with the maturation model developed by the model of Rhodin et al which was based on 923 subjects' data and several methods of GFR estimation. In this study, we also developed a maturation model from 93 subjects' data and this model's predictive power was also comparable with the predictive power of the maturation model proposed by Rhodin et al.

In this study, we also used an ADE model which has been proposed and validated from external data for the prediction of drug clearance from preterm neonates to adults (4). The ADE model uses different exponents for different age groups and is based on previously published allometric concepts $(38,39)$. The ADE models ability to predict GFR from preterm neonates to adults was as good as maturation or BDE models. The difference is that the ADE model is much easier to use than a maturation or BDE model.

The prediction of mean GFR from fixed exponent 0.75 (model 3) in preterm, term neonates, and younger children ( $\leq 3$ months of age) was the worst compared to other models. The substantial prediction error introduced by exponent 0.75 in GFR observed in this study is in line with the observations noted in other studies for the prediction of drug clearances in the aforementioned age groups $(4,38,40,41)$. In this study, however, it was observed that the predictive power of exponent 0.75 for GFR was comparable with other models in children $>1$ year of age (this will not be true for the prediction of drug clearances and generally exponent 0.75 provides a good prediction of drug clearances in children $>5$ years of age).

The inclusion of a sigmoidal model in the maturation model (Eq. 5) substantially improved the prediction of GFR in preterm and term neonates and infants as compared to the fixed exponent 0.75 . Therefore, the sigmoidal part of the maturation model serves as a correction factor to reduce the substantial error introduced by exponent 0.75 when used alone on body weight. As observed with the ADE model, it is evident that one will not require a correction factor if one does not use the exponent 0.75 across all age groups. It should also be noted that the benefit of sigmoidal part of the maturation model disappears after certain age and the prediction of GFR is dependent on body weight, adult GFR, and a fixed exponent of 0.75 . The parameters of maturation model are data dependent and are physiologically irrelevant $(4,42)$. Although the parameters of maturation model developed by Rhodin et al and the maturation model developed in this study are very different (due to differences in sample size and methods of GFR estimation in the model of Rhodin et $a l$ ), yet the predictive power of these two maturation models remains similar. The maturation model failed to predict individual GFR values within $\pm 30 \%$ in most of preterm and term neonates and infants.

Allometric models are generally developed using body weight and a parameter of interest. Generally, weight and age correlate well and there is no real benefit in developing an allometric model or in fact any mathematical model with two well correlated parameters (38). The maturation model uses both age and body weight; although there is no scientific basis 
to do so, it does help in reducing the substantial error introduced by exponent 0.75 in the prediction of drug clearance or GFR as seen in this study.

The allometric coefficients and exponents widely vary depending upon the sample size, body weight, or age. The exponents of allometry do not have any physiological or biological meaning. There is also no consensus if there is a scientific basis to use any fixed exponent across all age groups even though in many instances there are several allometric exponents embedded within these age groups (4). Recent studies by many investigators have shown that the concept of quarter-power law or so-called theoretical allometry (fixed exponents of $0.25,0.75$, or 1.0) is incorrect (43-51). The exponents of allometry for drug clearance in preterm and term neonates are generally $>1$ $(4,8-11,39)$. Therefore, it is not surprising that even for GFR the exponent 0.75 (model 3 ) provided a very poor prediction (systematic over-prediction by hundreds of percent) of GFR in preterm, term, and children $\leq 1$ year of age as compared to exponent 1.15, 1.0, or 0.9, respectively.

Unlike the fixed exponent 0.75 allometric model, the ADE model is based on different exponents for different age groups (as outlined in this study). Different exponents used in the ADE model substantially reduced prediction error in preterm, term, and children $\leq 1$ year of age as compared to a fixed exponent of 0.75 . The exponents used in the ADE model are from previous experience with drug clearances, BDE model in this study, and data analysis. It is important to note that the exponents used in the ADE model have no physiological meaning and are arbitrary. Furthermore, these exponents are not necessarily the best or optimum.

Several studies have indicated that a single allometric exponent does not describe the entire data (body weight versus drug clearance or volume of distribution) across all age groups $(4,8-11)$. This was demonstrated by some recently published population PK studies. The authors of these studies develop models based on allometric exponents which change with body weight (BDE model). The studies demonstrated the importance for using different exponents as a function of body weight to predict drug clearance across different age groups. The BDE models also negate the notion that age along with body weight is required to predict drug clearance in children. We borrowed this concept to develop a BDE model for GFR. Like the BDE models developed for the prediction of drug clearance across different age groups, the BDE model's predictive power for GFR was as good as ADE and maturation models. The BDE models differ from previous models (for clearance) in that the exponents of allometry for GFR also vary depending on body weight. In this study, the exponents of allometry in younger children were $>1$ and decreased with increasing age. This observation reconciles with the observations of the BDE models (8-11) as well as the nature of allometric exponents as observed by Brody, Wiesner, McMahon, and Bonner (52-54).

Overall, the predictive performance of the four models (BDE, ADE, and two maturation models) for the prediction of mean GFR was good but the prediction of GFR in individual healthy subjects was not very encouraging. In preterm and term neonates, the individual prediction error was quite high.
The limitations of the empirical models as described in this manuscript should be recognized and can be summarized as follows:

1. Although the main objective of this study and the model of Rhodin et al was to propose a simple model to predict renal function based on body weight and/or age and GFR, it appears that such a model is too simplistic to achieve the clinical objectives (dose selection based on predicted GFR). All models provided a good estimate of the population mean but failed to provide a reliable prediction of GFR in an individual subject. The practical application in the clinical settings of these models lies in individual rather than mean prediction of GFR. Biochemical markers such as creatinine or cystatin $\mathrm{C}$ concentrations may need to be incorporated along with age or weight in an empirical model to improve the prediction of GFR in an individual subject. Many such methods $(36,37)$ are currently in use with individual prediction error $\leq 30 \%$ in more than $80 \%$ subjects.

2. The empirical models as described in this manuscript were developed from healthy subjects' data and can only be used in healthy population. It is well known that the disease states such as renal impairment (in some cases hepatic impairment) can substantially influence the GFR. Critically ill patients may be with or without normal kidney function. In critically ill patients, if the kidney function is not compromised then these empirical models can be used but one must note that the predictive power of these models in an individual subject is not reliable. On the other hand, if the kidney function of the critically ill patients is compromised then these models should not be used at all. In fact, a model which predicts both normal and abnormal renal function in a population is more desirable than a model which predicts renal function only in healthy subjects. The empirical models described in this manuscript are not capable of detecting the magnitude of renal impairment; hence, these models cannot be used in disease state (renal impairment) which further limits the application of these models in clinical settings.

3. The data for the development of the models in this study as well as Rhodin et al study were obtained from studies conducted from 1940s to 1980s. Therefore, the measurement errors over time in the GFR values (especially in neonates and younger children) in association with the assay methodologies cannot be ignored. Same is true for the data used for model validation. Although mean GFR values for all age groups were predicted with fair degree of accuracy, the prediction of GFR in individual subjects was poor. The prediction error in the GFR values for individual subjects may be inflated to some extent due to the measurement errors and assay methodologies of these old data. However, the more likely explanation of the poor prediction of GFR in individual subjects is due to 
enormous variability across individual subject data (especially in preterm and term neonates) and it is difficult to capture this high variability from any empirical model (55). For example, in preterm and term neonates, the percent coefficient of variation $(\% \mathrm{CV})$ was 61 and $55 \%$, respectively. In adolescents and adults, \%CV was less than $20 \%$ and it was not surprising that the prediction of GFR in adolescents and adults from these models was superior to preterm and term neonates.

4. Measurement of creatinine concentrations and subsequently creatinine clearance may be influenced by age, muscle mass, and hydration state. For example, plasma creatinine concentrations at birth are much higher in relation to the size (and the muscle mass) of the newborn infant and remain so for 1 to 2 weeks (56). Especially, creatinine concentration is higher in preterm than term neonates and persists for a longer period of time. According to Guignard and Drukker (56), the high creatinine concentration levels in the newborn immediately after birth represent the maternal creatinine concentration and the continued high creatinine concentrations in preterm neonates may be due to tubular reabsorption. With time, total body muscle mass, GFR, and tubular secretion dictate the creatinine concentrations in an individual (56).

5. Since the individual prediction of GFR is erratic by the empirical models described in this manuscript, dose selection in an individual subject based on the predicted GFR by these models may not be used.

\section{CONCLUSIONS}

The current analysis indicates that the BDE, ADE, and maturation models can only predict mean GFR with accuracy in preterm and term neonates, infants, children, adolescents, and adults. Individual prediction of GFR in preterm and term neonates as well as in younger children is highly erratic and may be clinically unacceptable. This may not be surprising since all these models use only body weight (maturation model uses both body weight and age) which has no relationship with the true renal function of an individual. For example, the correlation coefficient $\left(r^{2}\right)$ between body weight and GFR (inulin clearance) for preterm neonates and adults was 0.307 $(n=109)$ and $0.001(n=34)$, respectively.

In this study, a $30 \%$ prediction error was considered clinically acceptable (based on the literature) but one should also evaluate if the prediction error of more than $30 \%$ in the GFR (for example 50\%) in an individual subject is clinically relevant?

It should be kept in mind that the models and formulas have their limits and, at present, there is no substitute for an accurate GFR determination (experimentally), particularly in neonates and young children. Models such as maturation, BDE, and allometric (and many others) may appear sophisticated on paper but in many instances the predictive power of these models may be poor and of little practical value to achieve certain clinical goals $(57,58)$. Therefore, it is of utmost importance that the models and equations are rigorously validated from external data before any practical use.

\section{REFERENCES}

1. Work DF, Schwartz GJ. Estimating and measuring glomerular filtration rate in children. Curr Opin Nephrol Hypertens. 2008; $17: 320-5$.

2. Rubin M, Bruck E, Rappoport M. Maturation of renal function in childhood clearance studies. J Clin Invest. 1949;28:1144-62.

3. Hayton WL. Maturation and growth of renal function: dosing renally cleared drugs in children. AAPS PharmSci 2000; 2:article $3(1-7)$.

4. Mahmood I, Staschen CM, Goteti K. Prediction of drug clearance in children: an evaluation of the predictive performance of several models. AAPS J. 2014;16:1334-43.

5. Anand KJ, Anderson BJ, Holford NH, NEOPAIN Trial Investigators Group, et al. Morphine pharmacokinetics and pharmacodynamics in preterm and term neonates: secondary results from the NEOPAIN trial. Br J Anaesth. 2008;101:680-9.

6. Anderson BJ. Pediatric models for adult target-controlled infusion pumps. Paediatr Anaesth. 2010;20:223-32.

7. Anderson BJ, Larsson P. A maturation model for midazolam clearance. Paediatr Anaesth. 2011;21(3):302-8.

8. Wang C, Sadhavisvam S, Krekels EH, et al. developmental changes in morphine clearance across the entire paediatric age range are best described by a bodyweight-dependent exponent model. Clin Drug Investig. 2013;33:523-34.

9. Wang C, Peeters MY, Allegaert K, et al. A bodyweightdependent allometric exponent for scaling clearance across the human life-span. Pharm Res. 2012;29:1570-81.

10. Wang C, Allegaert K, Peeters MY, Tibboel D, Danhof M, Knibbe CA. The allometric exponent for scaling clearance varies with age: a study on seven propofol datasets ranging from preterm neonates to adults. $\mathrm{Br} \mathrm{J}$ Clin Pharmacol. 2014;77:149-59.

11. Bartelink IH, Boelens JJ, Bredius RG, et al. Body weightdependent pharmacokinetics of busulfan in paediatric haematopoietic stem cell transplantation patients: towards individualized dosing. Clin Pharmacokinet. 2012;51:331-45.

12. Rhodin MM, Anderson BJ, Peters AM, Coulthard MG, Wilkins $\mathrm{B}$, Cole $\mathrm{M}$, et al. Human renal function maturation: a quantitative description using weight and postmenstrual age. Pediatr Nephrol. 2009;24:67-76.

13. Coulthard MG. Maturation of glomerular filtration in preterm and mature babies. Early Hum Dev. 1985;11:281-92.

14. Dean RFA, McCance RA. Inulin, diodone, creatinine and urea clearances in newborn infants. J Physiol. 1947;106:431-9.

15. Leake RD, Trygstad CW, Oh W. Inulin clearance in the newborn infant: relationship to gestational and postnatal age. Pediatr Res. 1976;10:759-62.

16. Aperia A, Broberger O, Thodenius K, Zetterström R. Development of renal control of salt and fluid homeostasis during the first year of life. Acta Paediatr Scand. 1975;64:393-8.

17. Barnett HL, Hare K, McNamara H, Hare R. Measurement of glomerular filtration rate in premature infants. J Clin Invest. 1948;27:691-9.

18. Vogelstein B, Kowarski A, Lietman PS. The pharmacokinetics of amikacin in children. J Pediatr. 1977;91:333-9.

19. Davies DF, Shock NW. Age changes in glomerular filtration rate, effective renal plasma flow, and tubular excretory capacity in adult males. J Clin Invest. 1950;29:496-507.

20. van der Heijden AJ, Grose WF, Ambagtsheer JJ, Provoost AP, Wolff ED, Sauer PJ. Glomerular filtration rate in the preterm infant: the relation to gestational and postnatal age. Eur J Pediatr. 1988;148:24-8.

21. Guignard JP, Torrado A, Da Cunha O, Gautier E. Glomerular filtration rate in the first three weeks of life. J Pediatr. $1975 ; 87: 268-72$. 
22. Leake RD, Trygstad CW. Glomerular filtration rate during the period of adaptation to extrauterine life. Pediatr Res. 1977;11(9 Pt 1):959-62.

23. Hedman A, Alván G, Strandvik B, Arvidsson A. Increased renal clearance of cefsulodin due to higher glomerular filtration rate in cystic fibrosis. Clin Pharmacokinet. 1990;18:168-75.

24. Hedman A, Adan-Abdi Y, Alvan G, Strandvik B, Arvidsson A. Influence of the glomerular filtration rate on renal clearance of ceftazidime in cystic fibrosis. Clin Pharmacokinet. 1988;15:57-65.

25. Paisley JW, Smith AL, Smith DH. Gentamicin in newborn infants. Comparison of intramuscular and intravenous administration. Am J Dis Child. 1973;126:473-7.

26. van den Anker JN, Pokorna P, Kinzig-Schippers M, Martinkova $\mathrm{J}$, de Groot R, Drusano GL, et al. Meropenem pharmacokinetics in the newborn. Antimicrob Agents Chemother. 2009;53:3871-9.

27. Wettrell G, Andersson KE, Bertler A, Lundström NR. Concentrations of digoxin in plasma and urine in neonates, infants, and children with heart disease. Acta Paediatr Scand. 1974;63(5):705-10.

28. Lanao JM, Dominguez-Gil AA, Dominguez-Gil A, Málaga S, Crespo M, Santos F. Pharmacokinetics of amikacin in children with normal and impaired renal function. Kidney Int. 1981;20:115-21.

29. Yoshioka H, Takimoto M, Matsuda I, Hattori S. Dosage schedule of gentamicin for chronic renal insufficiency in children. Arch Dis Child. 1978;53:334-7.

30. Wilson CB, Koup JR, Opheim KE, Adelman LA, Levy J, Stull TL, et al. Piperacillin pharmacokinetics in pediatric patients. Antimicrob Agents Chemother. 1982;22:442-7.

31. Winslade NE, Adelman MH, Evans EJ, Schentag JJ. Single-dose accumulation pharmacokinetics of tobramycin and netilmicin in normal volunteers. Antimicrob Agents Chemother. 1987;31:605-9.

32. Blouin RA, Kneer J, Stoeckel K. Pharmacokinetics of intravenous cefetamet (Ro 15-8074) and oral cefetamet pivoxil (Ro 158075 ) in young and elderly subjects. Antimicrob Agents Chemother. 1989;33(3):291-6.

33. Golper TA, Noonan HM, Elzinga L, Gilbert D, Brummett R, Anderson JL, et al. Vancomycin pharmacokinetics, renal handling, and nonrenal clearances in normal human subjects. Clin Pharmacol Ther. 1988;43:565-70.

34. Cutler NR, Narang PK, Lesko LJ, Ninos M, Power M. Vancomycin disposition: the importance of age. Clin Pharmacol Ther. 1984;36:803-10.

35. Evaluation of laboratory measurements for clinical assessment of kidney disease (part 5). In: National Kidney Foundation: K/DOQI clinical practice guidelines for chronic kidney disease: evaluation, classification, and stratification. Am J Kidney Dis. 2002; 39(Suppl 1): S76-S92.

36. Selistre L, De Souza V, Cochat P, Antonello IC, Hadj-Aissa A, Ranchin B, et al. GFR estimation in adolescents and young adults. J Am Soc Nephrol. 2012;23:989-96.

37. Schwartz GJ, Work DF. Measurement and estimation of GFR in children and adolescents. Clin J Am Soc Nephrol. 2009;4:183243.

38. Mahmood I. Prediction of clearance in children from adult clearance: allometric scaling versus exponent 0.75 . In: Pharmacokinetic allometric scaling in pediatric drug development. Rockville, MD: Pine House Publishers; 2013. pp. 41-55.

39. Mahmood I. Prediction of drug clearance in preterm and term neonates: different exponents for different age groups. In:
Pharmacokinetic allometric scaling in pediatric drug development. Rockville, MD: Pine House Publishers; 2013. pp. 88-109.

40. Peeters MY, Allegaert K, Blussé van Oud-Alblas HJ, et al. Prediction of propofol clearance in children from an allometric model developed in rats, children and adults versus a 0.75 fixedexponent allometric model. Clin Pharmacokinet. 2010;49:269-75.

41. Björkman S. Prediction of cytochrome p450-mediated hepatic drug clearance in neonates, infants and children: how accurate are available scaling methods? Clin Pharmacokinet. 2006;45:111.

42. Mahmood I. Evaluation of sigmoidal maturation and allometric models: prediction of propofol clearance in neonates and infants. Am J Ther. 2013;20:21-8.

43. Painter PR. The fractal geometry of nutrient exchange surfaces does not provide an explanation for 3/4-power metabolic scaling. Theor Biol Med Model. 2005;2:30.

44. Petit G, Anfodillo T. Plant physiology in theory and practice: an analysis of the WBE model for vascular plants. J Theor Biol. 2009;259:1-4.

45. Glazier DS. Beyond the '3/4-power law': variation in the intraand interspecific scaling of metabolic rate in animals. Biol Rev Camb Philos Soc. 2005;80:611-62.

46. White CR, Cassey P, Blackburn TM. Allometric exponents do not support a universal metabolic allometry. Ecology. 2007;88:315-23.

47. Packard GC, Birchard GF. Traditional allometric analysis fails to provide a valid predictive model for mammalian metabolic rates. J Exp Biol. 2008;211(Pt 22):3581-7.

48. Mahmood I. Theoretical versus empirical allometry: facts behind theories and application to pharmacokinetics. J Pharm Sci. 2010;99:2927-33.

49. West D, West BJ. Physiologic time: a hypothesis. Phys Life Rev. 2013;10:210-24.

50. Bentley LP, Stegen JC, Savage VM, et al. An empirical assessment of tree branching networks and implications for plant allometric scaling models. Ecol Lett. 2013;16:1069-78.

51. Glazier S. Metabolic scaling in complex living systems. Systems. 2014;2:451-540.

52. Brody S. Bioenergetics and growth, with special reference to the efficiency complex in domestic animals. London: Hafner Press, New York, and MacMillan Publishers; 1945.

53. Wieser W. A distinction must be made between the ontogeny and the phylogeny of metabolism in order to understand the mass exponent of energy metabolism. Respir Physiol. 1984;55:19.

54. McMohan TA, Bonner JT. Proportions and size. In: On size and life. New York: Scientific American Library; 1983. pp. 25-67.

55. Tayman C, Rayyan M, Allegaert K. Neonatal pharmacology: extensive interindividual variability despite limited size. J Pediatr Pharmacol Ther. 2011;16:170-84.

56. Guignard JP, Drukker A. Why do newborn infants have a high plasma creatinine? Pediatrics. 1999;103, e49.

57. Cella M, Zhao W, Jacqz-Aigrain E, Burger D, Danhof M, Della Pasqua O. Paediatric drug development: are population models predictive of pharmacokinetics across paediatric populations? $\mathrm{Br}$ J Clin Pharmacol. 2011;72:454-64.

58. Santen G, Horrigan J, Danhof M, Della Pasqua O. From trial and error to trial simulation. Part 2: an appraisal of current beliefs in the design and analysis of clinical trials for antidepressant drugs. Clin Pharmacol Ther. 2009;86:255-62. 